\title{
BLIND ADAPTIVE MULTIUSER DETECTION WITH PROBABILISTIC ALGORITHMS: APPLICATION TO UNDERWATER ACOUSTICS
}

\author{
Carles Antón-Haro ${ }^{1} \quad J o s e ́$ A.R. Fonollosa ${ }^{1} \quad$ Zoran Zvonar ${ }^{2} \quad$ Javier R. Fonollosa ${ }^{1}$ \\ ${ }^{1}$ Dpt. of Signal Theory and Communications - Universitat Politècnica de Catalunya. \\ c/ Gran Capità s/n. Campus Nord UPC. 08034 Barcelona (SPAIN). \\ e-mail: \{carles,adrian,fono\}@gps.tsc.upc.es \\ ${ }^{2}$ Analog Devices. Communications Division. \\ Wilmington MA 01887-1024 (USA). \\ e-mail: Zoran.Zvonar@analog.com
}

\begin{abstract}
In this paper ${ }^{1}$, two probabilistic adaptive algorithms for jointly detecting active users in a DS-CDMA system are compared. The first one, which is based on the theory of Hidden Markov Models (HMM) is proposed within the CDMA scenario and compared with the previously developed Viterbi-based algorithm. Both techniques are completely: blind in the sense that no knowledge of the signature sequences, channel state information or training sequences is required for any user. After convergence, an estimate of the signature of each user convolved with its physical channel inpulse response (CIR), and estimated data sequences are provided. This CIR estimate can then be used to switch to any decision-directed (DD) adaptation scheme. Performance of the algorithms is verified with simulations as well as with experimental data from an Underwater Acoustics (UWA) environment. In both cases, performance is found to be highly satisfactory, showing the near-far resistance of the analyzed algorithms.
\end{abstract}

\section{INTRODUCTION}

Recently, multiuser detection in CDMA systems has received increasing attention [1]. Detectors developed so far (conventional receiver, optimum detector, decorrelating and the AMSE detectors [2]) should know (or should be able to acquire) one or more of the parameters from the following list [3]:

1. The signature waveforms of the desired user and/or interfering users.

2. The timing (bit-epoch and carrier phase) of the desired user and/or interfering users.

3. The received amplitudes of the interfering users in relation $\pi^{* i t h}$ that of the desired user.

The MMSE detector is more suited for adaptive implementation on the basis of mean square error (MSE) minimization [1]. In that case, previous knowledge on interferers can be circumvented by making use of 4) training sequences, not only during the startup period but also after sudden changes in the channel impulse response (CIR) or when a new active user appears. The need to retransmit training sequences may be cumbersome in multiuser communications so that, in recent years, a large effort has been made in developing blind algorithms which perform CIR acquisition and data detection without such information (i.e. only on the basis of the channel output).

The algorithms presented and compared in this paper, are absolutely blind in the sense that no knowledge of 1)

${ }^{3}$ This work has been partially supported by the Fundació $\mathrm{Ca}$ talana per a la Recerca, the CIRIT (1996SGR-00096) (Generalitat de Catalunya) and the CICYT of Spain (Grants Nr. TIC951022-C05-1 and TIC96-0500-C10-01). to 4) is required for proper operation. They both belong to the group of Probabilistic algorithms $[4,5]$ and lead to joint channel estimation and data detection. These methods exhibit higher computational complexity but they outperform other blind methods (i.e. Bussgang and Polyspectra-based) since they make better use of the known statistical information on the input signal and, in general, require less symbols to obtain an accurate CIR estimate.

\section{SIGNAL MODEL}

We consider the general asynchronous multiple-access channel model in which the received signal is given by

$$
r(t)=\sum_{n} \sum_{k=1}^{K} b_{k}[n] h_{k}(t-n T)+\sigma w(t)
$$

where $h_{k}(t-n T)$ is the overall complex channel impulse response of user $k$, given by the convolution of its $M$-chip signature sequence, physical channel and receiving filter responses. For ease of notation, it incorporates the amplitude and the delay for user $k$ and its duration is assumed to be no longer than $L$ symbol periods. The total number of active users is $K$ and their transmitted data sequences are binary independent symbols $b_{k}[n] \in\{-1,1\}$. The symbol rate is $1 / T$ and $w(t)$ is normalized AWGN. The multipleaccess channel is sampled at a rate $f s=1 / T s=M / T$ to derive the discrete vector sequence $r[n]$ :

$$
\mathbf{r}[n]=\left[r(n T), \ldots, r\left(n T+(M-1) T_{s}\right)\right]^{T}
$$

where ${ }^{T}$ denotes transpose operation. The observation $r[n]$ can be modeled as a $M$-length vector, probabilistic function of the state vector $s[n]$ :

$$
r[n]=\mathbf{H}[n] s[n]+\mathbf{w}[n] .
$$

Since at any given time a maximum of $L$ symbols for each user affect the observation, there are $N=2^{K L}$ possible state vectors corresponding to all combinations of $L$ binary symbols of the $K$ active users. We denote each of the possible states as the $K L$-length vector $\mathbf{s}_{j}$,

$$
\mathbf{s}_{j} \in \mathcal{S}=\left\{\mathbf{s}_{1}, \mathbf{s}_{2}, \ldots, \mathbf{s}_{N}\right\}
$$

such that,

$$
\begin{aligned}
s_{j} & =\left[s_{j 1}^{T}, \ldots, s_{j K}^{T}\right]^{T}, \\
s_{j k} & =\left[s_{j k}^{(0)}, \ldots, s_{j k}^{(L-1)}\right]^{T}, s_{j k}^{(l)} \in\{1,-1\} .
\end{aligned}
$$

The actual state at time instant $n T$ is denoted by $s[n] \in S$. The $M \times K L$ matrix $\mathbf{H}[n]$ depends on the overall discrete impulse responses for each user, denoted by matrices $\mathbf{H}_{k}[n]$

$$
\mathbf{H}[n]=\left[\mathbf{H}_{1}[n], \ldots, \mathbf{H}_{K}[n]\right] \text {. }
$$


Each of these matrices incorporates a vector response for the $L$ symbols that may be present in the observation due to the ISI or to the asynchronous reception,

$$
\mathbf{H}_{k}[n]=\left[\mathbf{h}_{k 0}[n], \ldots, \mathbf{h}_{k(L-1)}[n]\right]
$$

and, finally, the resulting signature for each user $k$ and symbol $l$ :

$$
\mathbf{h}_{k l}[n]=\left[\begin{array}{c}
h_{k l}((n+l) T) \\
\vdots \\
h_{k l}\left((n+l) T+(M-1) T_{s}\right)
\end{array}\right] .
$$

The noise is characterized as the $M$-length vector $\mathrm{w}[n]$ :

$$
\mathrm{w}[n]=\sigma\left[w(n T), \ldots, w\left(n T+(M-1) T_{s}\right)\right]^{T} .
$$

This signal model is common to both the BW-based and the Viterbi-based algorithms and will be further refined to meet the formal requirements of each algorithm.

\section{BLIND IDENTIFICATION AND DETECTION ALGORITHMS}

We propose two algorithms on the basis of the Baum\&Welch (BW) reestimation procedure and the Viterbi algorithm. To achieve channel identification, both algorithms operate on a trellis-like structure.

\subsection{Multiuser Adaptive Baum\&Welch algorithm (MABW)}

The BW algorithm, which relies on the theory of Hidden Markov Models (HMM), is essentially identical to the EM method and it is known to lead, at least, to a local maximum of the likelihood function [6]. When dealing with timevarying channels, an adaptive version called $A B W$ (Adaptive Baum\&Welch) can be considered [7]. The probability, $\gamma_{j}[n]$, of being in state $j: 1 . . N=2^{K L}$ in the trellis at time instant $n$ given the sequence and the HMM is computed by means of the Forward-Backward algorithm [8]. The estimated multiuser CIR is updated at the symbol rate considering the steepest-descent adaptation scheme:

$$
\hat{\mathbf{H}}[n]=\hat{\mathbf{H}}[n-1]+\mu_{h} E\left[\mathbf{e}[n] \mathbf{s}[n]^{H}\right],
$$

where

$$
\mathbf{e}[n]=\mathbf{r}[n]-\mathbf{m}[n]=\mathbf{r}[n]-\hat{\mathbf{H}}[n] \mathbf{s}[n]
$$

and $\mu_{h}$ is the adaptation constant. In our blind environment, the expectation in the gradient term will be computed on the basis of the received sequence up to instant $n+\Delta$ (where the lag $\Delta$ is a design parameter taking typical values of 4-8 symbols), and the model at instant $n-1$ :

$$
\begin{aligned}
\hat{\mathbf{H}}[n] & =\hat{\mathbf{H}}[n-1]+\mu_{h} E_{r_{n+\Delta}, \lambda_{n-1}}\left[\mathbf{e}[n] \mathbf{s}[n]^{H}\right] \\
& =\hat{\mathbf{H}}[n-1]+\mu_{h} \sum_{j=1}^{N} \gamma_{j}[n] \mathbf{e}_{j}[n] \mathbf{s}_{j}^{H}
\end{aligned}
$$

and

$$
\mathbf{e}_{j}[n]=\mathbf{r}[n]-\mathbf{m}_{j}[n]=\mathbf{r}[n]-\hat{\mathbf{H}}[n-1] \mathbf{s}_{j} \quad j=1 . . N .
$$

Data detection is performed following an individually mostlikely state criterion [8].
3.2. Multiuser Adaptive Viterbi algorithm (MAV) This blind estimation algorithm [5] is initialized with an arbitrary CIR estimate for each state in the model. Note that, now, such CIR matrix contains as many matrices defined in Eq.(7) as the number of states. Namely, the $M \times K L N$ matrix:

$$
\begin{aligned}
\hat{\mathcal{H}}_{j}[n] & =\left.\hat{\mathbf{H}}[n]\right|_{\text {state }} j \\
\hat{\mathcal{H}}[n] & =\left[\hat{\mathcal{H}}_{1}[n], \ldots, \hat{\mathcal{H}}_{N}[n]\right] .
\end{aligned}
$$

Each estimate is updated following a stochastic-gradient (LMS) scheme that only takes into account the most likely preceding state among the $2^{K}$ predecessors:

$$
\hat{\mathcal{H}}_{j}[n]=\hat{\mathcal{H}}_{i}[n-1]+\mu_{h} \mathrm{e}_{i j}[n] \mathbf{s}_{j}^{H},
$$

where the state $s_{i}$ is the ML predecessor to state $s_{j}$, and the error vector $\mathbf{e}_{i j}[n]$ is defined as:

$$
\mathrm{e}_{i j}[n]=\mathbf{r}[n]-\hat{\mathcal{H}}_{i}[n-1] \mathbf{s}_{j} \quad j=1 . . N .
$$

In this second case, data is recovered following a most-likely state-sequence (MLSE) criterion.

\subsection{Considerations}

These two blind algorithms are well-suited for the startup period of a centralized receiver; after convergence, we can switch to a DD mode of operation (for example, a linear equalizer updated with a MMSE strategy [2]). Simulation results in [9], show that the CIR estimate obtained with the blind algorithm is good enough to make such change feasible.

As for computational complexity, the most-contributing section is computation of $\gamma_{i}[n]$ in the case of the MABW algorithm, whose counterpart for MAV is the computation of the metrics. Both contribute similarly to the total amount of operations $\left(O\left(N^{2}\right)\right)$ up to a multiplicative factor $\left(\Delta_{e q}+1\right)$ in the case of the MABW algorithm. This factor shows up as a consequence of the need of recursively (re)compute the backward variable [8] in the process of obtaining $\gamma_{i}[n]$. For example, if the backward variable is computed at each iteration step then $\Delta_{e q}=\Delta$. However, it is not an important drawback since this factor can be significantly reduced with the use of the sawtooth-lag scheme [10] where $\Delta_{e q}$, the equivalent lag factor, can be forced to be approximately equal to 1. Regarding the rest of sections, they all contribute on the order of $O(N)$ or less, and equally for both algorithms. As a final conclusion, the computational complexity of both strategies is approximately the same for a moderate number of users and low delay-spread, and on the same order of magnitude in any other case.

Whereas the MABW algorithm is (slightly) more costly in terms of number of operations per symbol, MAV demands more memory resources. This is a direct consequence of keeping track of CIRs separately (i.e. one estimate for each state) which increases memory requirement exponentially in the number of users and the duration of the CIR.

Finally, noting that when the multiuser signal is affected by the near-far effect, both algorithms tend to converge towards local maxima [5]. To overcome this problem, we adopted the strategy suggested in [5] called coherence checking.

\section{COMPARATIVE PERFORMANCE STUDY}

\subsection{Simulation Analysis}

The system under study operates at the symbol rate employing a BPSK modulation scheme. In all cases, $K=4$ users contributed to the CDMA signal. Gold sequences with $M=7$ chips were used as spreading sequences; coherence 


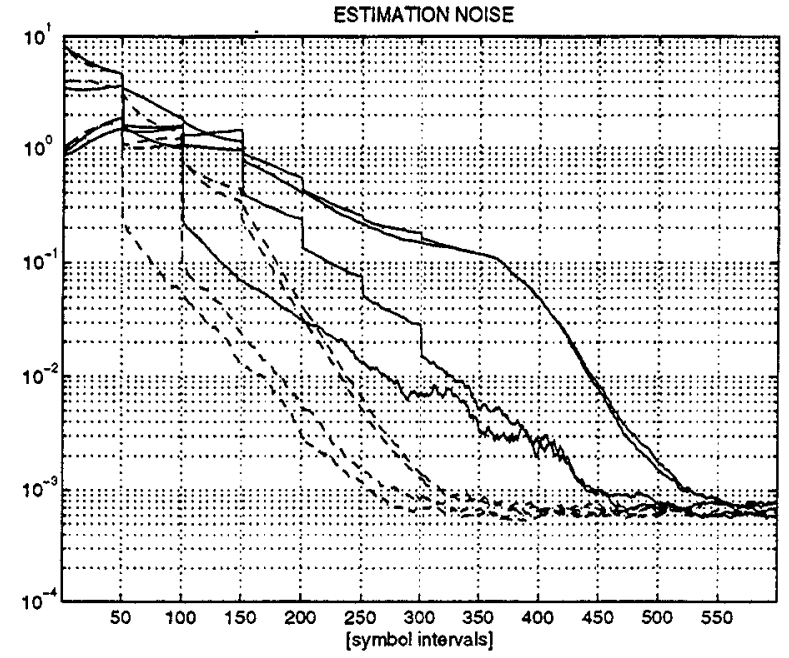

Figure 1. Learning curves. MABW (solid) and MAV (dash-dot). Amplitudes:3,2,1,1. SNR=12 dB.

checking were performed every $S=50$ symbols. A stationary and single-path model was considered for the channel. For all simulations, the incoming signal was sampled at the chip rate. Simulation results are averaged over 50 -run tests.

The estimation noise -learning curves- for all users and both algorithms are plotted in Fig.1. Such parameter is the euclidean distance between CIR and the corresponding estimate. A slower convergence for the MABW algorithm is observed. This fact is a direct consequence of the way in the CIR estimates are obtained: an average over all the possible present states (MABW) or, otherwise, an update of the CIR estimate corresponding to each state considering only the preceding state (MAV). Within first iteration steps, when there is not a predominant path in the trellis, all states contribute to update the CIR estimate; this extent guarantees convergence of MABW at least towards a local minimum but, of course, brings down convergence speed.

We also note that, despite of the joint detection strategy, users are extracted sequentially according to their power level.

Regarding to estimation noise in the final steady state, similar levels for both algorithms are observed. In fact, when convergence has been achieved, it is absolutely equivalent to have a single averaged CIR estimate with no contribution from states different from the most likely (MABW), or to have a very predominant state whose CIR estimate will be chosen as the most likely at each instant (MAV).

In Fig.2, we plot the response of both systems in an environment with very low SNR (6dB, for the weakest user). Users separation is also achieved at the expense of a slightly slower convergence rate for the weakest users in the MABW algorithm. Behaviour when facing strong near-far effect (approx. $30 \mathrm{~dB}$ ) is depicted in Fig.3. Convergence is faster now since, as long as special measures were adopted to overcome near-far effect (coherence checks), the difference in the received amplitudes helps the algorithm to distinguish between signals coming from different users. To conclude this section, the algorithms were tested considering asynchronous reception (Fig.4). Delays considered (wrt the first user) were 1, 2 and 3 chips. We do not observe significant changes in the behaviour with respect to the synchronous case. Note, however, that the number of states is four times higher since double the number of taps are required for the CIR estimate.

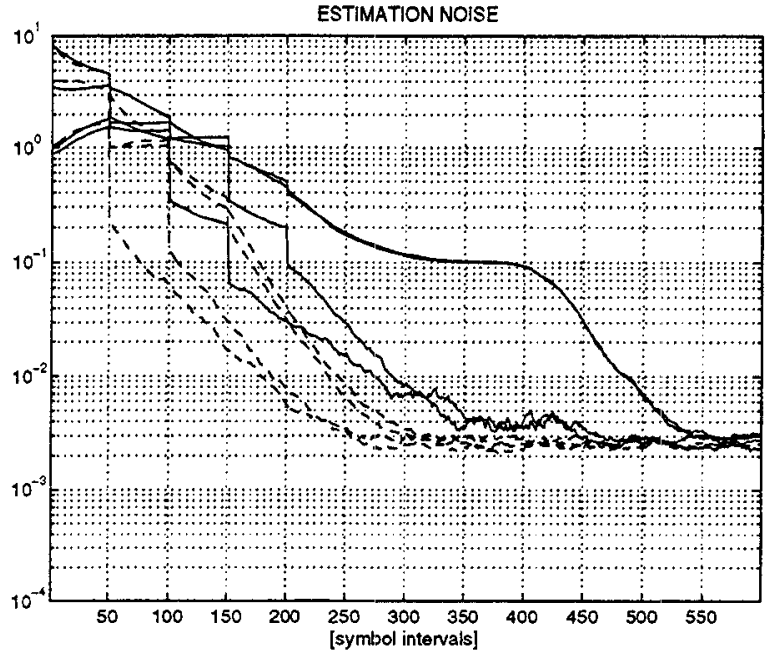

Figure 2. Learning curves in a very low SNR environment: MABW (solid) and MAV (dash-dot). Amplitudes: $3,2,1,1$. SNR $=6 \mathrm{~dB}$.

\subsection{Evaluation with Experimental Data}

Further validation of the algorithms has also been carried out by considering experimental data obtained in a Underwater Acoustic (UWA) environment from a vertical link established between acoustic modems. Two users contributed to the CDMA signal (signatures: $[1,1,1]$ and $[1,-1,1]$; gaussian shaping pulses). The SNR was on the order of 20 $\mathrm{dB}$ and the difference in received power between users 10 $\mathrm{dB}$ (approx.). ISI was negligible (see [11] for details).

We chose the span of the estimated CIR to be equal to $L=2$ symbols and an oversampling factor of 4 (4 samples/chip). The rest of pararneters were assigned the same values as those mentioned before. Results for the MABW algorithm are plot in Fig.5. First, we observe that the estimates for signatures and amplitudes (time $n=550$ ) corresponding to both users match the ones described above. Convergence is achieved within the first 150 symbols after one coherence compensation at $n=50$. Only 2 and 21 errors were observed respectively in the whole packet. Similar results were obtained with the MAV algorithm.

\section{CONCLUSIONS}

In this paper, two probabilistic algorithms for JD of DSCDMA signals and channel estimation have been presented and compared. The MABW algorithm is based on the theory of Hidden Markov Models, whereas the MAV is a blind version of the well-known Viterbi algorithm. Both algorithms are adaptive and the estimate of the convolution of each user's signature with the physical channel is recursively updated using gradient schemes. Nevertheless, since the receiver operates blindly, training sequences are replaced by estimates of the transmitted data based on the received signal and the present estimate of the parameter set.

As a consequence of algorithmic differences, the MABW algorithm is slightly more computationally intensive but, in contrast, memory requirements are less strict than those of MAV. Simulation study indicates that both algorithms exhibit a very similar behaviour. They only differ in convergence time which is higher in the case of MABW. In turn, this assures the convergence of this algorithm, at least, to a local maximum of the likelihood function. Performance of the MABW algorithm has also been verified on experimental data obtained in an UWA environment. 


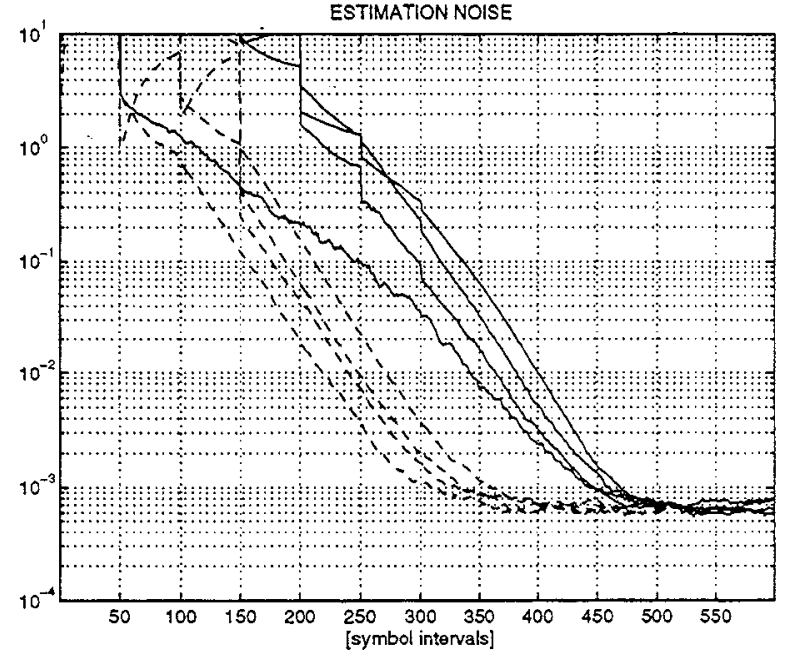

Figure 3. Learning curves with near-far effect $(30$ dB): MABW (solid) and MAV (dash-dot). Amplitudes: $30,7,5,1$. SNR $=12 \mathrm{~dB}$.

Both algorithms are well-suited for the startup period of a centralized multiuser receiver; after convergence, switching to a less computationally-intensive DD adaptation method would be advisable.

Undergoing research focuses on algorithm extension to include array observation. Further analysis is, mainly, application specific, including tests with synthetic signals generated by standard test channels (such as those proposed in the GSM recommendations) and exhaustive tests with experimental data both in radio and UWA environments.

\section{REFERENCES}

[1] S. Verdú, "Adaptive multiuser detection," in Proc. IEEE Intl. Symp. on Spread Spectrum Techniques, (Oulu (Finland)), pp. 43-50, 1994.

[2] J.G. Proakis, Digital Communications. McGraw Hill, 3rd edition ed., 1995.

[3] M. Honig, U. Madhow, and S. Verdú, "Blind adaptive multiuser detection," IEEE Trans. on Information Theory, vol. IT-41, pp. 944-960, July 1995.

[4] M. Ghosh and C.L. Weber, "Maximum likelihood blind equalization," Proc. SPIE Adaptive Signal Processing, vol. 1565 , pp. 188-195, 1991.

[5] J.R. Fonollosa, J.A.R. Fonollosa, Z. Zvonar, and J. Vidal, "Blind multiuser identification and detection in CDMA systems," in Proc. IEEE Int. Conf. Acoust. Speech and Signal Processing, (Detroit (USA)), pp. 1876-1879, May 1995.

[6] L.E. Baum, T. Petrie, G. Soules, and N. Weiss, "A maximization technique ocurring in the statistical analysis of probabilistic functions of markov chains," The Annals of Mathematical Statistics, vol. 41 , no. 1, pp. 164171,1970 .

[7] J.A.R. Fonollosa and J. Vidal, "Application of hidden markov models to blind channel characterization and data detection," in Proc. IEEE Int. Conf. Acoust. Speech and Signal Processing, (Australia), pp. 185-188, April 1994.

[8] L. Rabiner, "A tutorial on hidden markov models and selected applications in speech recognition," Proc. of the IEEE, vol. 77, pp. 257-286, Feb. 1989.

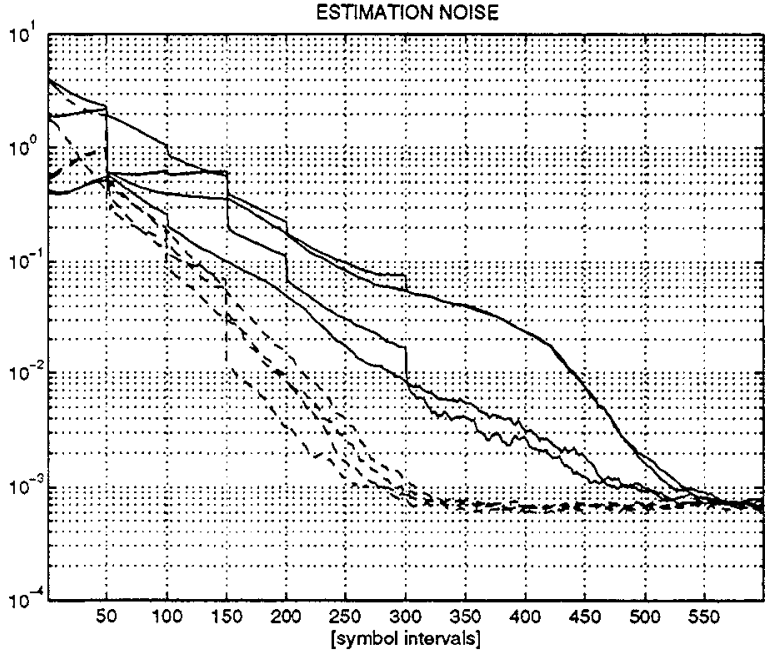

Figure 4. Learning curves in an asynchronous case: MABW (solid) and MAV (dash-dot). Amplitudes: $3,2,1,1$. Delays: $0,1,2,3$ chips. $S N R=12 \mathrm{~dB}$.
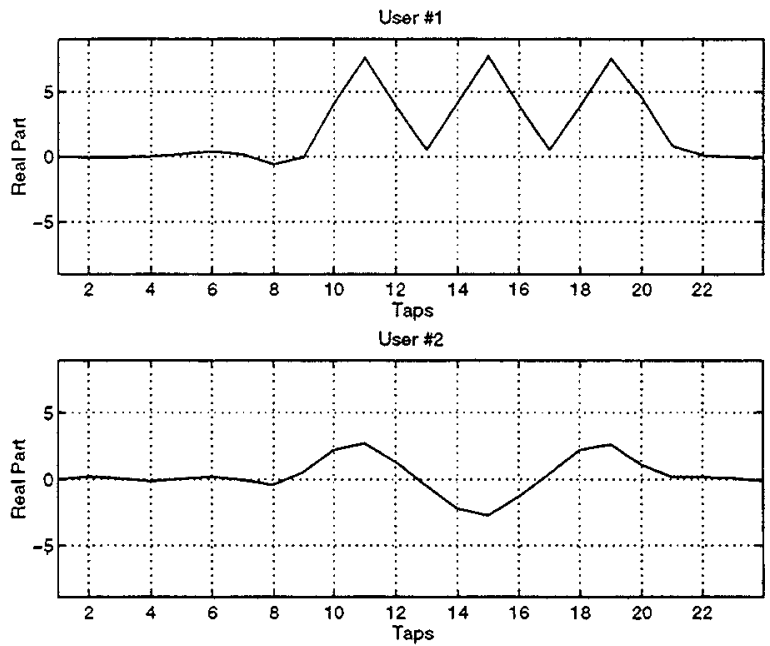

Figure 5. MABW algorithm CIR estimates for the UWA data. Amplitudes: 8,3. SNR $=20 \mathrm{~dB}$.

[9] C. Antón-Haro, J.A.R. Fonollosa, Z. Zvonar, and J.R. Fonollosa, "Probabilistic algorithms for blind adaptive multiuser detection" Submitted to IEEE Trans. on Signal Processing, 1996.

[10] V. Krishnamurthy and J. Moore, "On-line estimation of hidden markov model parameters based on the kullback-leibler information measure," IEEE Trans. on Signal Processing, vol. 41, pp. 2557-2573, Aug. 1993.

[11] Z. Zvonar, D. Brady, and J. Catipovic, "An adaptive linear multiuser receiver for deep water acoustic local area networks," in Proc. IEEE Int. Conf. Acoust. Speech and Signal Processing, vol. II, (Australia), pp. 389392, April 1994. 